\title{
lonosphere-weighted undifferenced and uncombined PPP-RTK: theoretical models and experimental results
}

\author{
Jiuping Zha ${ }^{1,2}$ (C) Baocheng Zhang ${ }^{1} \cdot$ Teng Liu $^{1} \cdot$ Pengyu Hou ${ }^{1,2}$
}

Received: 8 October 2020 / Accepted: 30 July 2021

(c) The Author(s) 2021

\begin{abstract}
Precise ionospheric information, as like precise satellite orbits, clocks, and code/phase biases, is a critical factor for achieving fast integer ambiguity resolution in precise point positioning (PPP-AR). This study develops an ionosphere-weighted (IW) undifferenced and uncombined PPP real-time kinematic (PPP-RTK) network model using code and phase observations. We introduce between-station single-differenced ionospheric delay pseudo-observations to take advantage of the similar characteristics of ionospheric delays between two receivers tracking the same satellite. The estimable ionospheric parameters are commonly affected by the differential code bias referring to a particular receiver assigned as pivot, which facilitates the ionospheric interpolation at the user side. Then, the kinematic positioning performance of the IW PPP-RTK user model is analyzed and compared with those of PPP-AR without ionospheric corrections, RTK, and IW-RTK models during low and high solar activity days. The results show that for the PPP-RTK model, the positioning errors converge to thresholds of $2 \mathrm{~cm}$ for the horizontal components and $5 \mathrm{~cm}$ for the vertical component within 20 epochs, and the positioning errors become stable after an initialization of 20 epochs with root-mean-squared (RMS) values of approximately $0.47,0.58$ and $1.66 \mathrm{~cm}$ for the east, north and up components, respectively, which are superior to those of the other three models. Owing to the high ionospheric disturbance influence, the RMS values of the east and up components increase by approximately double and the mean time-to-first-fix increases by $61.5 \%$ for the PPP-RTK case.
\end{abstract}

Keywords Ionosphere-weighted model $\cdot$ Integer ambiguity resolution $\cdot$ PPP-RTK $\cdot$ Kinematic positioning $\cdot$ Ionospheric disturbance

\section{Introduction}

Precise point positioning (PPP) proposed by Malys and Jensen (1990) and Zumberge et al. (1997) has advantages such as a high level of flexibility without the limitation of dense networks, supporting unidirectional or broadcast communication and requiring a low bandwidth with statespace representative corrections. However, a long convergence time of several tens of minutes is required to achieve decimeter-level accuracy (Van et al. 2009). The PPP realtime kinematic (PPP-RTK) is proposed by Wübbena et al. (2005) to improve the positioning accuracy and convergence

Baocheng Zhang

b.zhang@whigg.ac.cn

1 State Key Laboratory of Geodesy and Earth's Dynamics, Innovation Academy for Precision Measurement Science and Technology, Wuhan, China

2 University of Chinese Academy of Sciences, Beijing, China behavior. Ge et al. (2008) achieved the integer ambiguity resolution in PPP (PPP-AR) based on satellite phase biases without ionospheric constraints. Ionospheric delays are one of the most important error sources in GNSS measurements, and many unknown parameters must be estimated or corrected for precise GNSS applications. When the ionospheric products, besides the precise satellite orbit/clock errors and code/phase biases, are used to correct or constrain the GNSS observation equations, the float ambiguities are quickly fixed to integers, which ultimately improves the positioning accuracy and convergence behavior (Teunissen et al. 2010). Recently, the horizontal accuracy of sub- $10 \mathrm{~cm}$ is achieved within 6 min by Psychas and Verhagen (2020) at the user given a reference network with interstation distances of approximately $68 \mathrm{~km}$. It takes approximately $10.5 \mathrm{~min}$ to achieve sub-decimeter horizontal accuracy in a reference network with interstation distances of approximately $115 \mathrm{~km}$. 
In PPP-RTK processing, the network-derived ionospheric corrections are biased by the linear combination of receiver and satellite differential code biases (DCBs) as described by Teunissen et al. (2010) and Li et al. (2011). Three popular methods are used to avoid the influence of receiver DCBs in ionospheric spatial interpolation. First, only those satellites tracked simultaneously by all of the reference stations are involved in the calculation of the network corrections, which reduces the number of available satellites in practice when the reference network increases ( $\mathrm{Li}$ et al. 2011). Second, Psychas et al. (2018) used the thin-layer assumption and generalized triangular series function to isolate the ionospheric total electron contents (TECs) and DCBs, but the misspecification and mapping function errors decreased the accuracy of the precise ionospheric corrections ( $\mathrm{Li}$ et al. 2017). Third, user ionospheric delays are estimated using all of the network-derived ionospheric corrections and the betweenstation single-differenced ionospheric delays (Teunissen and Khodabandeh 2013). This processing adds additional calculation to the user side, and a similar processing step needs to be carried out repeatedly for all users. In addition, during storm-level ionospheric activity, the ionospheric spatial and temporal variations are significant, which can decrease the accuracy of ionospheric delay estimation and interpolation (Próchniewicz and Walo 2012). However, most previous studies were performed under low/medium ionospheric disturbance (Wang et al. 2017; Psychas and Verhagen 2020).

Over the past few years, several popular PPP-RTK methods, such as the integer recovery clock model (Laurichesse et al. 2009), the decoupled satellite clock model (Collins et al. 2008), the uncalibrated phase delay model (Ge et al. 2008), and the undifferenced and uncombined (UDUC) model (Teunissen et al. 2010) have been proposed. Compared with the former three ionosphere-free PPP-RTK methods, the UDUC PPP-RTK network model simultaneously estimates the ionospheric delays, which are the key for fast integer $\mathrm{AR}$ and are convenient for multi-frequency applications. Therefore, based on the UDUC PPP-RTK network model, we develop an ionosphere-weighted (IW) UDUC PPP-RTK network model under the constraint of between-station single-differenced ionospheric delays. The ionospheric pseudoobservations can strengthen the PPP-RTK network model. Also, the estimable ionospheric parameters contain the same receiver DCB of the network, instead of different receivers of the network, which is beneficial for user ionospheric interpolation. Then, the kinematic positioning performance of the IW UDUC PPP-RTK user model is analyzed. To understand the positioning performance improvement brought by the network ionospheric corrections, the results of the PPP-AR model are also analyzed. Similarly, the RTK and IW-RTK models are used to analyze the contribution of the betweenstation single-differenced ionospheric pseudo-observations, which are also used in the IW UDUC PPP-RTK network model. Finally, low and high solar activity conditions are considered in our experiment to analyze the influence of ionospheric disturbance, as the PPP-RTK network the user models are constrained by extra ionospheric delays.

\section{Methodology}

This section first reviews the UDUC observation equations to briefly introduce the observations and parameters involved in this study. Then, the IW UDUC PPP-RTK network model is developed from the UDUC PPP-RTK network model described by Odijk et al. (2016). Similarly, The UDUC PPP-RTK user model is given according to the PPPRTK network model.

\section{UDUC observation equations}

GPS code and phase observations are the basic data used in this study. The UDUC observed-minus-computed observation equations are given as (Teunissen and Montenbruck 2017):

$$
\left\{\begin{array}{c}
E\left(\Delta p_{r, j}^{s}(i)\right)=c_{r}^{s}(i) \cdot \Delta x_{r}(i)+c\left[d t_{r}(i)-d t^{s}(i)\right] \\
\quad+g_{r}^{s}(i) \cdot \tau_{r}(i)+\mu_{j} l_{r}^{s}(i)+d_{r, j}-d_{j}^{s} \\
E\left(\Delta \phi_{r, j}^{s}(i)\right)=c_{r}^{s}(i) \cdot \Delta x_{r}(i)+c\left[d t_{r}(i)-d t^{s}(i)\right] \\
\quad+g_{r}^{s}(i) \cdot \tau_{r}(i)-\mu_{j} l_{r}^{s}(i)+\delta_{r, j}-\delta_{j}^{s}+\lambda_{j} N_{r, j}^{s}
\end{array}\right.
$$

where $E(\cdot)$ denotes the expectation operator; $\Delta p_{r, j}^{s}(i)$ and $\Delta \phi_{r, j}^{s}(i)$ denote the code and phase observations, respectively, from satellite $s(s=1 \cdots m)$ tracked by receiver $r(r=1 \cdots n)$ at epoch $i ; m$ and $n$ represent the numbers of satellites and receivers; the subscript $j(j=1 \cdots f)$ denotes the frequency index, and $f$ represents the number of frequencies; the $3 \times 1$ vector $\Delta x_{r}(i)$ denotes the receiver's position increment; the $1 \times 3$ vectors $c_{r}^{s}(i)$ denotes the receiverto-satellite unit direction vector; $c$ denotes the velocity of light in vacuum; $d t_{r}(i)$ and $d t^{s}(i)$ denote the receiver and satellite clock errors, respectively; $\tau_{r}(i)$ denotes the zenith tropospheric wet delays; $g_{r}^{s}(i)$ denotes the mapping function of the tropospheric wet components; $l_{r}^{S}(i)$ denotes the firstorder slant ionospheric delay experienced on the first frequency $f_{1}$, where $\mu_{j}=f_{1}^{2} / f_{j}^{2}$ is the frequency-dependent multiplier factor; $d_{r, j}$ and $d_{j}^{s}$ denote the receiver and satellite code biases, respectively; $\delta_{r, j}$ and $\delta_{j}^{s}$ denote the receiver and satellite phase biases, respectively; $\lambda_{j}=c / f_{j}$ denotes the phase wavelength on frequency $j ; N_{r, j}^{s}$ denotes ambiguities.

The receivers experience more or less parallel line-of-sight vectors to the same satellite when the interstation distances 
are smaller than approximately $500 \mathrm{~km}$ (Rocken et al. 1993). Therefore, the tropospheric mapping functions are assumed to be equal for all the reference stations and expressed as $g_{r}^{s}(i)=g_{1}^{s}(i),(r=1 \cdots n)$. The satellite positions are calculated with precise satellite orbit products. The positions of network reference stations are fixed to a priori known value and the positions of user stations are estimated in the epochwise kinematic mode. The receiver and satellite code/phase biases and the ambiguities are treated as time-invariant parameters.

\section{IW UDUC PPP-RTK network model}

Equation (1) is not a full-rank system due to the linear dependency of some columns of the design matrix. Odijk et al. (2016) identified the null space of the design matrix and chose a minimum constraint set ( $S$-basis) to eliminate the rank deficiencies among those parameters. Ten types of rank deficiencies and the corresponding $S$-basis constraints are listed in Table 1 . In Table 1, $p$ and $q$ represent the pivot receiver and satellite, respectively; $d_{I F}^{s}, d_{G F}^{s}, d_{r, I F}$ and $d_{r, G F}$ are given as follows:

$$
\left\{\begin{array}{c}
d_{I F}^{s}=\frac{\mu_{2} d_{1}^{s}-\mu_{1} d_{2}^{s}}{\mu_{2}-\mu_{1}}, d_{G F}^{s}=\frac{d_{2}^{s}-d_{1}^{s}}{\mu_{2}-\mu_{1}}, d_{D C B}^{s}=d_{2}^{s}-d_{1}^{s} \\
d_{r, I F}=\frac{\mu_{2} d_{r, 1}-\mu_{1} d_{r, 2}}{\mu_{2}-\mu_{1}}, d_{r, G F}=\frac{d_{r, 2}-d_{r, 1}}{\mu_{2}-\mu_{1}}, d_{D C B}=d_{r, 2}-d_{r, 1}
\end{array}\right.
$$

When the rank deficiencies in (1) are eliminated according to the above $S$-basis, the full-rank UDUC PPP-RTK network code and phase observation equations (Odijk et al. 2016) are given as: where the estimable forms of the biased parameters represented by the - symbol are listed in Table 2 .

Equation (3) ignores the spatial correlation in the regional ionospheric delays. However, the slant ionospheric delays experienced by all involved receivers from the same satellite are approximately equal with a mutual distance of a few hundred kilometers (Odijk 2002). Therefore, the following extra observation equation (Wang et al. 2017) can be added to (1):

$0=l_{p}^{s}(i)-l_{r \neq p}^{s}(i), \quad W=S^{-1}$

where $W$ and $S$ denote the weight and variance-covariance matrixes, respectively, of the between-station singledifferenced ionospheric pseudo-observations. The variance-covariance matrix is further given as:

$S=c_{l}^{2}\left[\begin{array}{cccc}r_{11} & r_{12} & \cdots & r_{1 n} \\ r_{21} & r_{22} & \cdots & r_{2 n} \\ \vdots & \vdots & \ddots & \vdots \\ r_{n 1} & r_{n 2} & \cdots & r_{n n}\end{array}\right] \otimes\left[\begin{array}{cccc}c_{1}^{2} & 0 & \cdots & 0 \\ 0 & c_{2}^{2} & \cdots & 0 \\ \vdots & \vdots & \ddots & \vdots \\ 0 & 0 & \cdots & c_{m}^{2}\end{array}\right]$

with

$r_{a b}=\exp \left(-\left(\frac{h_{a b}}{h_{0}}\right)^{2}\right), c_{s}^{2}=\frac{1}{\sin ^{2}\left(E^{s}\right)},\left\{\begin{array}{c}a, b=1 \cdots n \\ s=1 \cdots m\end{array}\right.$

where $h_{a b}$ represents the interstation distance between stations $a$ and $b ; h_{0}=200 \mathrm{~km}$ represents the empirical reference interstation distance; $E^{s}$ denotes the elevation angle of satellite $s ; \otimes$ denotes the Kronecker product; and $c_{l}=0.3 \mathrm{~m}$

$\left\{\begin{array}{c}E\left(\Delta p_{r, j}^{s}(i)\right)=c\left[\bar{d} t_{r \neq p}(i)-\bar{d} t^{s}(i)\right]+g_{1}^{s}(i) \cdot \bar{\tau}_{r \neq p}(i)+\mu_{j} \bar{l}_{r}^{s}(i)+\bar{d}_{r \neq p, j>2}-\bar{d}_{j>2}^{s} \\ E\left(\Delta \phi_{r, j}^{s}(i)\right)=c\left[\bar{d} t_{r \neq p}(i)-\bar{d} t^{s}(i)\right]+g_{1}^{s}(i) \cdot \bar{\tau}_{r \neq p}(i)-\mu_{j} \bar{l}_{r}^{s}(i)+\bar{\delta}_{r \neq p, j}-\bar{\delta}_{j}^{s}+\lambda_{j} N_{r \neq p, j}^{s \neq q}\end{array}\right.$

Table 1 Ten rank deficiency types with the involved parameters, size, and S-basis for the PPP-RTK network observation equations

\begin{tabular}{llll}
\hline Type & Involved parameters & Size & S-basis \\
\hline $\mathrm{A}$ & $d t_{r}(\mathrm{i}) \& d t^{s}(i)$ & 1 & $d t_{p}(i)$ \\
$\mathrm{B}$ & $d_{r, j} \& d_{j}^{s}$ & $\mathrm{f}$ & $d_{p, j}$ \\
$\mathrm{C}$ & $\delta_{r, j} \& \delta_{j}^{s}$ & $\mathrm{f}$ & $\delta_{p, j}$ \\
$\mathrm{D}$ & $d t_{r \neq p}(i) \& d_{r \neq p, j} \& \delta_{r \neq p, j}$ & $\mathrm{n}-1$ & $d_{r \neq p, I F}$ \\
$\mathrm{E}$ & $d t^{s}(i) \& d_{j}^{s} \& \delta_{j}^{s}$ & $\mathrm{~m}$ & $d_{I F}^{s}$ \\
$\mathrm{~F}$ & $\delta_{j}^{s} \& N_{r, j}^{s}$ & $f \cdot m$ & $N_{p, j}^{s}$ \\
$\mathrm{G}$ & $\delta_{r \neq p, j} \& N_{r \neq p, j}^{s}$ & $f \cdot(n-1)$ & $N_{r \neq p, j}^{q}$ \\
$\mathrm{H}$ & $\tau_{r}(i) \& d t^{s}(i)$ & 1 & $\tau_{p}(i)$ \\
$\mathrm{I}$ & $l_{r}^{s}(i) \& d_{r \neq p, j} \& \delta_{r \neq p, j}$ & $\mathrm{n}-1$ & $d_{r \neq p, G F}$ \\
$\mathrm{~J}$ & $l_{r}^{s}(i) \& d_{j}^{s} \& \delta_{j}^{s}$ & $\mathrm{~m}$ & $d_{G F}^{s}$ \\
\hline
\end{tabular}

Table 2 Estimable parameters of the UDUC PPP-RTK network model

\begin{tabular}{ll}
\hline Parameter & Formulation \\
\hline $\bar{d}^{s}(i)$ & $d t^{s}(i)-d t_{p}(i)+\left[d_{I F}^{s}-d_{p, I F}-g_{1}^{s}(i) \cdot \tau_{p}(i)\right] / c$ \\
$\bar{d}_{j>2}^{s}$ & $d_{j}^{s}-d_{I F}^{s}-\mu_{j} d_{G F}^{s}-d_{p, j}+d_{p, I F}+\mu_{j} d_{p, G F}$ \\
$\bar{\delta}_{j}^{s}$ & $\delta_{j}^{s}-d_{I F}^{s}+\mu_{j} d_{G F}^{s}-\delta_{p, j}+d_{p, I F}-\mu_{j} d_{p, G F}-\lambda_{j} N_{p, j}^{s}$ \\
$\bar{N}_{r \neq p, j}^{s \neq q}$ & $\left(N_{r, j}^{s}-N_{p, j}^{s}\right)-\left(N_{r, j}^{q}-N_{p, j}^{q}\right)$ \\
$\bar{\tau}_{r \neq p}(i)$ & $\tau_{r}(i)-\tau_{p}(i)$ \\
$\bar{d}_{r \neq p}(i)$ & $d t_{r}(i)-d_{p}(i)+\left(d_{r, I F}-d_{p, I F}\right) / c$ \\
$\bar{d}_{r \neq p, j>2}$ & $\left(d_{r, j}-d_{r, I F}-\mu_{j} d_{r, G F}\right)-\left(d_{p, j}-d_{p, I F}-\mu_{j} d_{p, G F}\right)$ \\
$\bar{\delta}_{r \neq p, j}$ & $\left(\delta_{r, j}-d_{r, I F}+\mu_{j} d_{r, G F}+\lambda_{j} N_{r, j}^{q}\right)-\left(\delta_{p, j}-d_{p, I F}+\mu_{j} d_{p, G F}+\lambda_{j} N_{p, j}^{q}\right)$ \\
$\bar{l}_{r}^{s}(i)$ & $l_{r}^{s}(i)+d_{r, G F}-d_{G F}^{s}$ \\
\hline
\end{tabular}


denotes a priori precision of the between-station single-differenced ionospheric pseudo-observations.

As the between-station single-differenced ionospheric pseudoobservations are added, (1) no longer has the I-th type rank deficiency. After eliminating the rest of the rank deficiencies, the fullrank IW UDUC PPP-RTK network model is formulated as follows: receiver code bias of the network pivot receiver is included in all estimable ionospheric parameters. This means that the interpolated user ionospheric delays include the same receiver DCBs, which the user receiver code/phase bias estimates will eventually absorb.

$$
\left\{\begin{array}{c}
E\left(\Delta p_{r, j}^{s}(i)\right)=c\left[\bar{d} t_{r \neq p}(i)-\bar{d} t^{s}(i)\right]+g_{1}^{s}(i) \cdot \bar{\tau}_{r \neq p}(i)+\mu_{j} \bar{l}_{r}^{s}(i)+\bar{d}_{r \neq p, j>2}-\bar{d}_{j>2}^{s}+\frac{\mu_{j}}{\mu_{2}-\mu_{1}} \bar{d}_{r \neq p, D C B} \\
E\left(\Delta \phi_{r, j}^{s}(i)\right)=c\left[\bar{d} t_{r \neq p}(i)-\bar{d} t^{s}(i)\right]+g_{1}^{s}(i) \cdot \bar{\tau}_{r \neq p}(i)-\mu_{j} \bar{l}_{r}(i)+\bar{\delta}_{r \neq p, j}-\bar{\delta}_{j}^{s}+\lambda_{j} \bar{N}_{r \neq p, j}^{s \neq q} \\
0=\bar{l}_{p}^{s}-\bar{l}_{r \neq p}^{s}
\end{array}\right.
$$

where the estimable forms of the biased receiver phase biases and ionospheric delays are different from those in Table 2 and are listed in Table 3. More importantly, the between-receiver DCBs $d_{p r, D C B}$ are estimable.

As shown in Table 2, the estimable ionospheric parameters are biased by geometry-free receiver and satellite code biases that are also interpolated and contained in the user ionospheric delays. Therefore, when different combinations of network reference receivers track some satellites, the user ionospheric delays for different satellites will contain different combinations of network receiver code biases, which causes system biases in user positions. However, this is not a problem for the IW UDUC PPP-RTK network model. In Table 3, the geometry-free

Table 3 Estimable parameters for the IW UDUC PPP-RTK network model compared with Table 2

\begin{tabular}{ll}
\hline Parameter & Formulation \\
\hline $\bar{d}_{r \neq p, D C B}$ & $d_{r, D C B}-d_{p, D C B}$ \\
$\bar{\delta}_{r \neq p, j}$ & $\left(\delta_{r, j}-d_{r, I F}+\lambda_{j} N_{r, j}^{q}\right)-\left(\delta_{p, j}-d_{p, I F}+\lambda_{j} N_{p, j}^{q}\right)$ \\
$\bar{l}_{r}^{s}(i)$ & $l_{r}^{s}(i)+d_{p, G F}-d_{G F}^{s}$ \\
\hline
\end{tabular}

\section{UDUC PPP-RTK user model}

In theory, the user station can be treated as one part of the reference network. The types of rank deficiencies and corresponding $S$-bases in the user model are similar to those in the network model. The differences are that the user positions are estimated and $\bar{d} t^{s}(i), \bar{d}_{j>2}^{s}$, and $\vec{\delta}_{j}^{s}$ are corrected and moved to the left of (3) or (7). Therefore, the IW UDUC PPP-RTK user model is given as follows:

$\left\{\begin{array}{l}E\left(\Delta p_{u, j}^{s}(i)+c \bar{d} t^{s}(i)+\bar{d}_{j>2}^{s}\right) \\ \quad=c_{u}^{s}(i) \cdot \Delta x_{u}(i)+c \bar{d} t_{u}(i)+g_{u}^{s}(i) \cdot \bar{\tau}_{u}(i)+\mu_{j} \widehat{l}_{u}^{s}(i)+\widehat{d}_{u, j>2}+\mu_{j} \widehat{d}_{u, G F} \\ E\left(\Delta \phi_{u, j}^{s}(i)+c \bar{d} t^{s}(i)+\bar{\delta}_{j}^{s}\right) \\ \quad=c_{u}^{s}(i) \cdot \Delta x_{u}(i)+c \bar{d} t_{u}(i)+g_{u}^{s}(i) \cdot \widehat{\tau}_{u}(i)-\mu_{j} \widehat{l}_{u}^{s}(i)+\widehat{\delta}_{u, j}+\lambda_{j} \widehat{N}_{u, j}^{s \neq q} \\ \hat{l}_{\text {Interpolate }}^{s}(i)=\widehat{l}_{u}(i)\end{array}\right.$

where the estimable forms of the biased parameters represented by the --symbol are listed in Table 4 . When there are no ionospheric constraints, (8) represents the PPP-AR model, and $\vec{d}_{u, G F}$ does not exist. Similarly, when the interpolated network ionospheric corrections $\vec{l}_{\text {Interpolate }}^{-s}(i)$ are used as pseudo-observations on the user side, (8) represents the
Table 4 Estimable parameters for the PPP-AR and PPP-RTK models

\begin{tabular}{lll}
\hline Parameter & PPP-AR & PPP-RTK \\
\hline $\bar{d} t_{u}(i)$ & $\left(d t_{u}(i)+d_{u, I F} / c\right)-\left(d t_{p}(i)+d_{p, I F} / c\right)$ & \\
$\bar{\tau}_{u}(i)$ & $\tau_{u}(i)-g_{1}^{s}(i) / g_{u}^{s}(i) \cdot \tau_{p}(i)$ & \\
$\hat{N}_{u, j}^{s \neq q}$ & $\left(N_{u, j}^{s}-N_{u, j}^{q}\right)-\left(N_{p, j}^{s}-N_{p, j}^{q}\right)$ & \\
$\hat{d}_{u, j>2}$ & $\left(d_{u, j}-d_{u, I F}-\mu_{j} d_{u, G F}\right)-\left(d_{p, j}-d_{p, I F}-\mu_{j} d_{p, G F}\right)$ & \\
$\bar{d}_{u, G F}$ & no & $d_{u, G F}-d_{p, G F}$ \\
$\hat{l}_{u}(i)$ & $l_{u}^{s}(i)-d_{G F}^{s}+d_{u, G F}$ & $l_{u}^{s}(i)-d_{G F}^{s}+d_{p, G F}$ \\
$\widehat{\delta}_{u, j}$ & $\left(\delta_{u, j}-d_{u, I F}+\lambda_{j} N_{u, j}^{q}+\mu_{j} d_{u, G F}\right)$ & $\left(\delta_{u, j}-d_{u, I F}+\lambda_{j} N_{u, j}^{q}\right)$ \\
& $-\left(\delta_{p, j}-d_{p, I F}+\lambda_{j} N_{p, j}^{q}+\mu_{j} d_{p, G F}\right)$ & $-\left(\delta_{p, j}-d_{p, I F}+\lambda_{j} N_{p, j}^{q}\right)$ \\
\hline
\end{tabular}


PPP-RTK model, and $\widehat{d}_{u, G F}$ must be estimated. In the PPPRTK case, the variance factors of the network-derived ionospheric delays are also interpolated as a priori precision of the ionospheric pseudo-observations.

In addition, the RTK and IW-RTK models described by Odolinski et al. (2015) are used to provide comparisons for the PPP-AR and PPP-RTK models. In the IW-RTK model, ionospheric pseudo-observations are used based on (4) and (5). The positions of the reference stations have been fixed to a priori known values to provide absolute user positions.

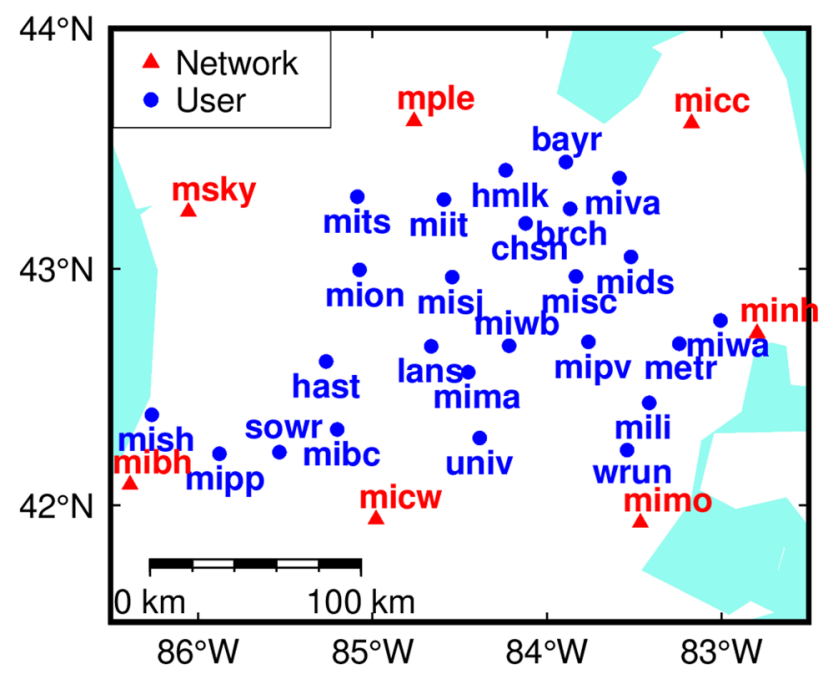

Fig. 1 Overview of the experimental reference and user stations

\section{Experimental setup}

The experimental datasets were composed of GPS dual-frequency code and phase observations with a $30 \mathrm{~s}$ sampling rate provided by 32 stations, namely 7 network reference stations and 25 user stations from the Missouri CORS network (Fig. 1). The mean interstation distance between two adjacent reference stations is approximately $117.5 \mathrm{~km}$. To carry out the RTK experiment, 25 baselines were made up of user stations and their nearest reference stations with mean interstation distances of approximately $59.8 \mathrm{~km}$. Data from days 251/252/253 in 2014 and days 254/255/256 in 2018 were selected. Days 251/252/253 in 2014 are high solar activity days with an average sunspot number of 166 and an X-class flare, and days 254/255/256 in 2018 are low solar activity days with an average sunspot number of 10 .

The main data processing strategies are listed in Table 5. The Kalman filter is reinitialized every $4 \mathrm{~h}$ on the user side. The first receiver of the reference network is selected as the pivot receiver because all receivers are equivalent, and the satellite with a maximum elevation angle for each station is used as the pivot satellite. In addition, the bootstrapped success rate with a threshold of $99.99 \%$ is used to choose the float ambiguities that are further imported into the leastsquares ambiguity decorrelation adjustment (LAMBDA) method (Teunissen 1995) to achieve partial AR (PAR). The fixed-failure rate ratio (FFRatio) test was applied to judge whether the ambiguities are successfully fixed to the integers under a threshold value obtained by lookup tables with a failure rate of $0.1 \%$ (Teunissen and Verhagen 2009). When the data pass the FFRatio test, we can obtain ambiguity-resolved

Table 5 Data processing strategy used in this study

\begin{tabular}{ll}
\hline Items & Strategies \\
\hline Frequency & GPS L1\&L2 \\
Estimator & Kalman filter \\
Weighting strategy & Elevation-dependent weighting \\
Priori precision & Code observations: $0.3 \mathrm{~m} ;$ \\
& Phase observations: $0.3 \mathrm{~cm}$ \\
Tropospheric delays & A priori value provided by UNB3m; \\
& Estimated as random-walk noise 0.001 m; \\
& GMF is used as the mapping function \\
Ionospheric delays & Network: estimated as white noise; \\
& User: estimated as white noise or constrained by the network corrections using Kriging interpolation \\
& (Olea 1999); \\
Elevation cutoff angles & Network: 8 degrees; \\
& User: 10 degrees \\
Detection of the cycle slip & Geometry-free single-channel detection identification adaptation approach (Odijk and Verhagen 2007) \\
Ambiguity & Network: estimated as float constants for each arc; \\
P1-C1 satellite DCB & User: PAR \\
\hline
\end{tabular}


positioning solutions called fixed solutions. For PARs, only when enough ambiguities, for example, more than $60 \%$ of all the float ambiguities in our study, are successfully fixed can the positioning solutions stabilize and achieve a high level of accuracy. Therefore, epochs when more than $60 \%$ of all float ambiguities are successfully fixed are used to calculate the time-to-first-fix (TTFF) and the empirical fixed rate $P_{\text {fixed }}$.

\section{Results and analysis}

In this section, the kinematic positioning performance of the PPP-RTK model, including the convergence behavior, TTFF, fixed rate, and positioning accuracy, is analyzed and compared with those of the PPP-AR, IW-RTK and RTK models on days with low solar activity. Then, the regional ionospheric TECs estimated by the IW UDUC PPP-RTK network model during high and low solar activity days are presented and analyzed. Finally, the positioning performances of the four models during high solar activity days are compared with those during low solar activity days.

\section{Positioning performance during the low solar activity days}

The kinematic positioning performance of 25 stations on three successive days is analyzed in detail for four PAR cases during low solar activity days. Before that, as the ionosphere delays exhibit a diurnal variation ( $\mathrm{Li}$ et al. 2015), the typical time series of the kinematic positions for the PPP-RTK are shown in Fig. 2. As shown by the blue dots, there is a convergence time for the fixed solutions because the number of fixed ambiguities gradually increases. When more than $60 \%$ of all float ambiguities are successfully fixed to integers, as indicated by the red arrow, the positioning errors become stable with absolute values less than $2 \mathrm{~cm}$ for the horizontal components and $5 \mathrm{~cm}$ for the vertical component. As shown by the green dots, the formal errors, especially for the east component, agreed with the positioning errors. When the formal errors of the east component converge to $2 \mathrm{~cm}$, the positioning errors become stable. Therefore, the formal error can be used as an important index to evaluate the positioning performance when there are no a priori known values for user positions. The positioning performances of the fixed solutions in Fig. 2 are further exhibited in Table 6. Clearly, the positioning performances during 12:00 to 14:00 UTC are superior to those during 16:00 to $18: 00 \mathrm{UTC}$, mainly because the ionospheric disturbance is strong during 10:00

Table 6 Positioning performances of the fixed solutions exhibited in Fig. 2. The RMS values are calculated from the 30th minutes for each 2-h session

\begin{tabular}{llllcc}
\hline $\begin{array}{l}\text { UTC } \\
\text { (hour) }\end{array}$ & \multicolumn{2}{l}{ RMS $(\mathrm{cm})$} & TTFF (min) & $\begin{array}{l}P_{\text {fixed }} \\
(\%)\end{array}$ \\
\cline { 2 - 5 } & East & North & Up & & \\
\hline $12: 00 \sim 14: 00$ & 0.4 & 0.4 & 1.1 & 8.0 & 93.3 \\
$16: 00 \sim 18: 00$ & 0.4 & 0.4 & 2.0 & 12.0 & 90.0 \\
\hline
\end{tabular}

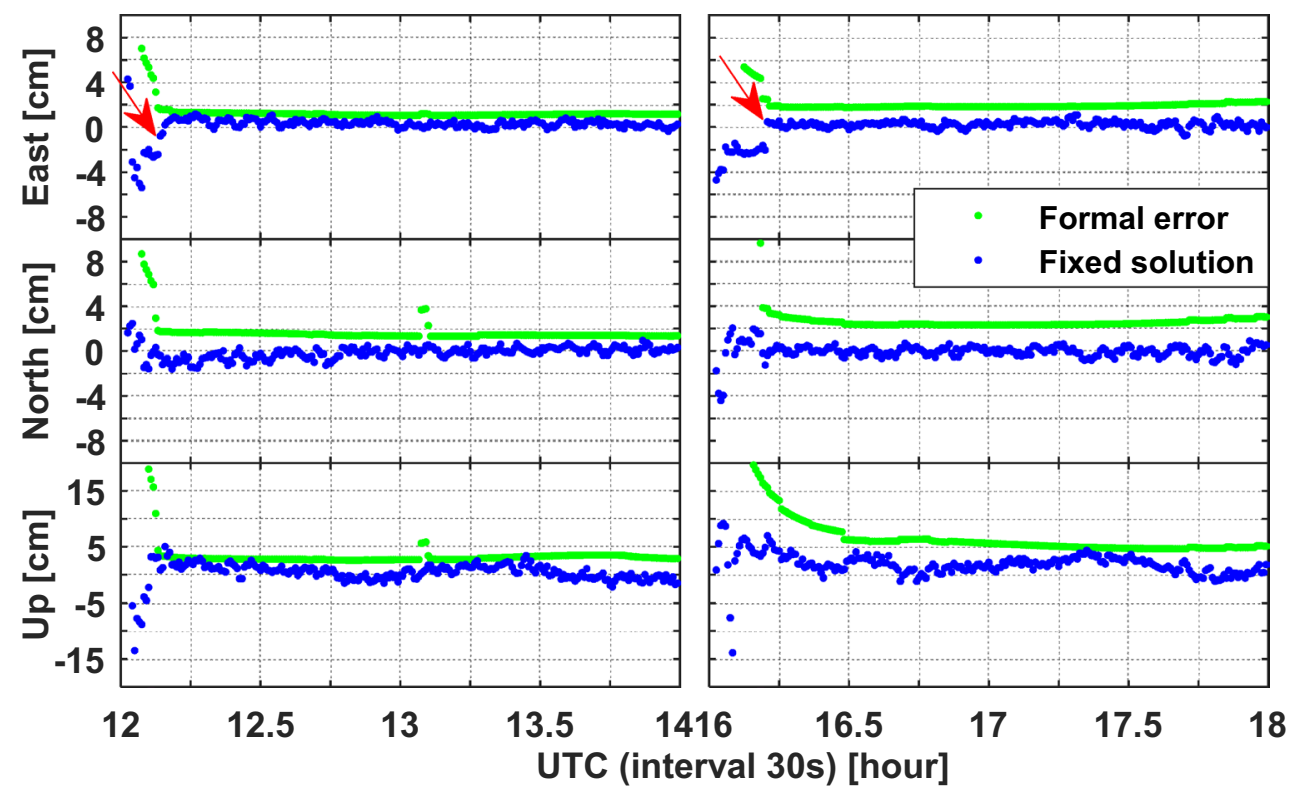

Fig. 2 Time series of the kinematic user positions (relative to the ground truth) estimated by the PPP-RTK model at station BAYR on day 254 in 2018 
to 18:00 local time in one day which corresponds to $16: 00$ to 24:00 UTC in Missouri (Li et al. 2015).

Figure 3 depicts the convergence behavior of the positioning errors of all the 4-h sessions for the 25 user stations. As shown by the upper-left and upper-right panels, the positioning performance of the float solutions of the PPP-RTK model is inferior to those of the other three models. However, the positioning performance of the fixed solutions of the PPP-RTK model is superior to those of the other three models during the whole convergence period, as shown by the lower three panels. The principal reason is that the PPP-RTK model is strongly constrained by the interpolated network ionospheric corrections, while between-station single-differenced ionospheric delays weakly constrain the IW-RTK model, and the PPP-AR and RTK models are not constrained directly by extra ionospheric delays. The interpolation model limits the precision of the interpolated ionospheric pseudo-observations. During the early period of convergence, the interpolated ionospheric pseudo-observations increase the number of available observations, which accelerates the convergence of the user parameters. When the float solutions of the PPPRTK model converge to a certain precision, such as $5 \mathrm{~cm}$ for the east component or $20 \mathrm{~cm}$ for the up component,

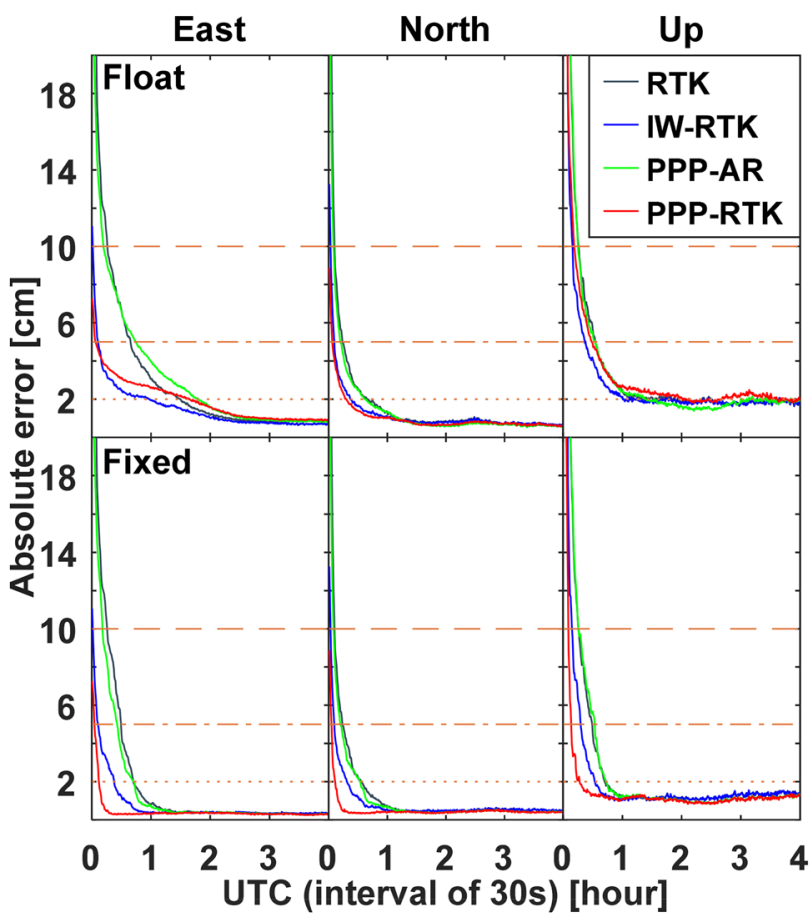

Fig. 3 Convergence behavior of the float-ambiguity (upper three panels) and fixed-ambiguity (lower three panels) positioning errors of all the 4-h sessions for the 25 user stations on days 254/255/256 in 2018 . The dashed, dash-dotted, and dotted lines denote the thresholds of $10 \mathrm{~cm}, 5 \mathrm{~cm}$, and $2 \mathrm{~cm}$, respectively the contribution of the interpolated network ionospheric pseudo-observations weakens. However, for the fixed solutions of the PPP-RTK model, the interpolated ionospheric pseudo-observations accelerate the convergence of user parameters. The variance factors of the satellite clock errors and phase biases and the interpolated ionospheric variance factors are conducive to quickly obtaining the correct integer ambiguity using the LAMBDA method because of the high cross-correlation between satellite clock errors, satellite phase biases, and ionospheric parameters.

Table 7 shows the mean convergence time for thresholds of $10 \mathrm{~cm}, 5 \mathrm{~cm}$, and $2 \mathrm{~cm}$. The convergence time is equal to $0.5 \mathrm{~min}$ in the horizontal direction, which means that the mean horizontal positioning errors are less than $10 \mathrm{~cm}$ from the first epoch. In addition, the mean positioning errors converge to $2 \mathrm{~cm}$ for the horizontal components and $5 \mathrm{~cm}$ for the vertical component within $10 \mathrm{~min}$ in the PPP-RTK case. With thresholds of $2 \mathrm{~cm}$ in the horizontal components and $5 \mathrm{~cm}$ in the vertical component, the mean convergence time for the east, north, up components is reduced by $92.4 \%, 61.1 \%, 70.7 \%$ for the PPP-RTK case, $60.8 \%, 13.3 \%, 0.0 \%$ for the PPP-AR case, $60.7 \%$, $15.6 \%, 18.2 \%$ for the IW-RTK case, and $52.8 \%, 16.5 \%$, $10.6 \%$ for the RTK case, respectively, from the float- to fixed-ambiguity solutions. The integer nature of ambiguity mainly improves the convergence behavior in the east component (Ge et al. 2008; Blewitt 1989). Furthermore, network-derived precise ionospheric corrections are used in the PPP-RTK model, which leads to an improvement of more than $50 \%$ in the convergence time in the east, north and up components.

Table 8 lists the mean RMS values of the ambiguityfixed positions from the 10th minute or the 90th minute of each 4-h session. In the PPP-RTK case, the mean RMS value of the positioning errors from the 10th minute is close to that from the 90th minute, which means that the positioning accuracy in horizontal and vertical directions is at millimeter and centimeter level from the 10th minute. But the positioning accuracies of the other three cases are improved greatly when the time threshold is changed from the 10th minute to the 90th minute. Even so, the mean RMS values from the 90th minute are close for the four cases. The mean TTFF for the PPP-RTK case is approximately $10 \mathrm{~min}$, which is less than one-third that of the other three cases. In addition, above $95 \%$ of all epochs are the high-accuracy ambiguity-fixed position solutions for the PPP-RTK case, while below $90 \%$ are for the other three cases, and most of the float solutions are at the initial period for all cases. All of these results demonstrate the superiority of the PPP-RTK model for achieving fast PAR compared with the other three models. In addition, 
Table 7 Mean convergence times for thresholds of $2 \mathrm{~cm}$, $5 \mathrm{~cm}$, and $10 \mathrm{~cm}$ for all the 4-h sessions of the 25 user stations on days 254/255/256 in 2018

\begin{tabular}{|c|c|c|c|c|c|c|c|c|}
\hline & \multicolumn{3}{|c|}{ East (min) } & \multicolumn{3}{|c|}{ North (min) } & \multicolumn{2}{|c|}{$\mathrm{Up}(\min )$} \\
\hline & $2 \mathrm{~cm}$ & $5 \mathrm{~cm}$ & $10 \mathrm{~cm}$ & $2 \mathrm{~cm}$ & $5 \mathrm{~cm}$ & $10 \mathrm{~cm}$ & $5 \mathrm{~cm}$ & $10 \mathrm{~cm}$ \\
\hline & \multicolumn{8}{|l|}{ Float } \\
\hline PPP-RTK & 99.0 & 4.0 & 0.5 & 18.0 & 4.0 & 0.5 & 29.0 & 11.5 \\
\hline PPP-AR & 108.5 & 45.0 & 12.0 & 37.5 & 12.0 & 5.5 & 32.5 & 15.5 \\
\hline IW-RTK & 58.5 & 6.5 & 1.5 & 22.5 & 6.0 & 2.0 & 22.0 & 9.5 \\
\hline RTK & 90.0 & 39.5 & 16.0 & 39.5 & 14.5 & 6.0 & 33.0 & 16.0 \\
\hline \multicolumn{9}{|l|}{ Fixed } \\
\hline PPP-RTK & 7.5 & 3.0 & 0.5 & 7.0 & 3.0 & 0.5 & 8.5 & 5.5 \\
\hline PPP-AR & 42.5 & 26.0 & 11.0 & 32.5 & 12.0 & 5.5 & 32.5 & 15.5 \\
\hline IW-RTK & 23.0 & 6.5 & 1.5 & 19.0 & 6.0 & 2.0 & 18.0 & 9.5 \\
\hline RTK & 42.5 & 30.0 & 16.0 & 33.0 & 14.5 & 6.0 & 29.5 & 16.0 \\
\hline
\end{tabular}

Table 8 Mean RMS values, TTFFs and fixed rates $P_{\text {fixed }}$ of the positioning errors from the 10th minutes or the 90th minutes for all the 4-h sessions of the 25 user stations on days $254 / 255 / 256$ in 2018

\begin{tabular}{llllll}
\hline & \multicolumn{2}{l}{$\begin{array}{l}\text { Overall mean RMS } \\
(>10 \mathrm{~min} />90 \text { min, unit: } \mathrm{cm})\end{array}$} & TTFF (min) & $\begin{array}{l}P_{\text {fixed }} \\
(\%)\end{array}$ \\
\cline { 2 - 4 } & East & North & Up & & \\
\hline PPP-RTK & $0.5 / 0.4$ & $0.6 / 0.6$ & $1.7 / 1.4$ & 10.4 & 95.5 \\
PPP-AR & $2.9 / 0.5$ & $1.7 / 0.6$ & $4.3 / 1.5$ & 38.8 & 82.9 \\
IW-RTK & $1.1 / 0.5$ & $1.1 / 0.7$ & $2.6 / 1.7$ & 30.4 & 87.0 \\
RTK & $3.7 / 0.5$ & $2.1 / 0.7$ & $4.1 / 1.7$ & 43.3 & 81.6 \\
\hline
\end{tabular}

the mean TTFF for the IW-RTK case is less than that of the RTK case, and the mean fixed rate for the IW-RTK case is higher than that of the RTK case, which reflects indirectly that the between-station single-differenced ionospheric pseudo-observations are beneficial for the IW UDUC PPP-RTK network model.

\section{Regional ionospheric TECs during the low and high solar activity days}

Figure 4 shows the regional ionospheric TECs estimated by the IW UDUC PPP-RTK network model for high and low solar activity days. The minimum of the regional ionospheric TECs has been subtracted from the raw networkderived ionospheric TECs to give an idea of the horizontal ionospheric variation. Clearly, both the absolute and relative ionospheric TECs for the high solar activity day are larger than those for the low solar activity day. In our study, the between-station single-differenced ionospheric delays are used as pseudo-observations in the network model, which may be affected by high ionospheric disturbance. In addition, the Kriging interpolation method is used to obtain the user ionospheric delays from the network-derived ionospheric corrections, which are also affected by high ionospheric disturbance. Therefore, it is necessary to analyze further the influence of high ionospheric disturbance on the positioning performances of the PPP-RTK, PPP-AR, IWRTK, and RTK models.
Fig. 4 Network-derived ionospheric TECs (unit: TECU) at 6 moments on the high solar activity day (upper panels) and low solar activity day (lower panels). The minimum of the regional ionospheric TECs has been subtracted from the raw network-derived TECs and marked in parentheses. All panels have the same $\mathrm{X}$-axis range of $-95.0^{\circ} \sim-72.5^{\circ}$ and the same Y-axis range of $33.0^{\circ} \sim 48.0^{\circ}$

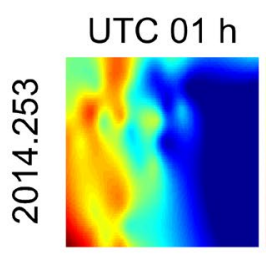

(11.1)

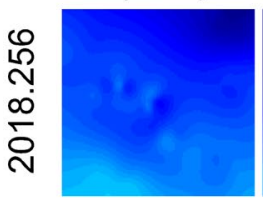

(4.0)
$05 \mathrm{~h}$

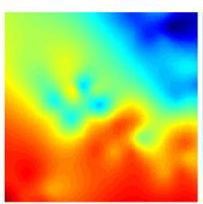

(2.2)

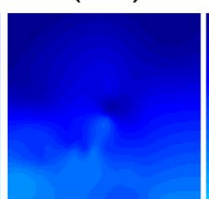

$(1.3)$
$09 \mathrm{~h}$

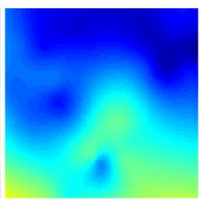

(3.7)

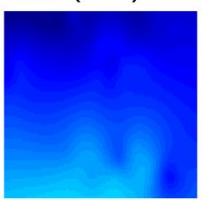

(0.9)
$13 \mathrm{~h}$

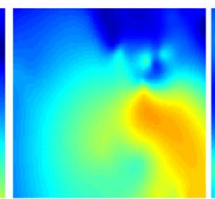

(17.3)

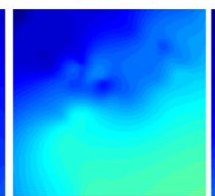

(3.0)
$17 \mathrm{~h}$

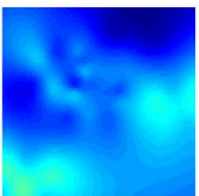

(25.1)

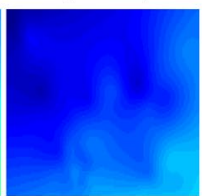

(6.5)
$21 \mathrm{~h}$

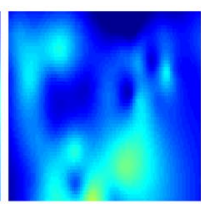

(30.7)

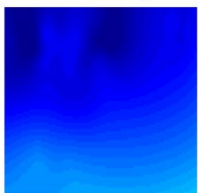

(6.8)

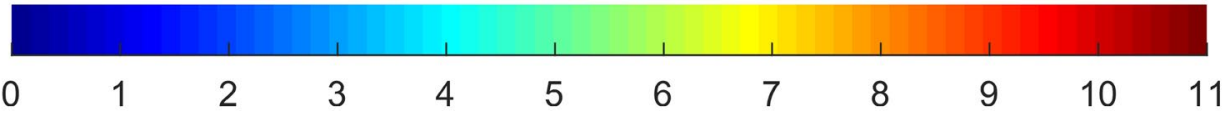




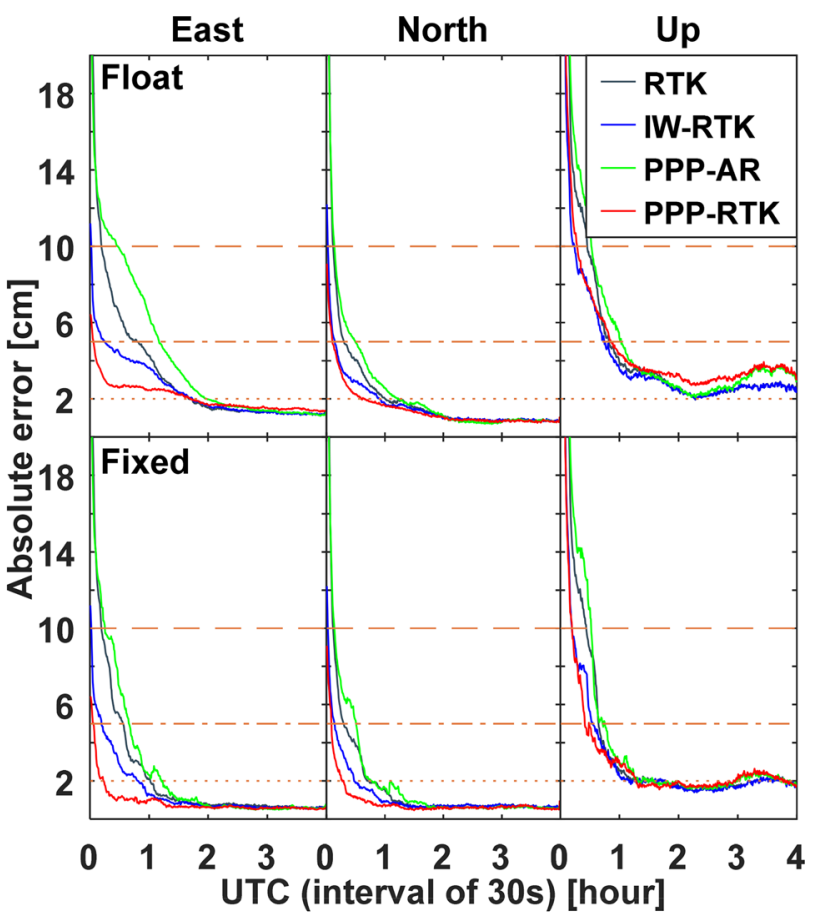

Fig. 5 Convergence behavior of the float- (top panels) and fixedambiguity (bottom panels) positioning errors of all the 4-h sessions for the 25 user stations on days $251 / 252 / 253$ in 2014. The dashed, dash-dotted, and dotted lines denote the convergence thresholds of $10 \mathrm{~cm}, 5 \mathrm{~cm}$, and $2 \mathrm{~cm}$, respectively

\section{Positioning performance during the high solar activity days}

Figure 5 shows the convergence behavior during the high solar activity days. Obviously, the convergence behaviors in Fig. 5 are worse than those in Fig. 3, meaning that the high ionospheric disturbance has decreased the positioning performance considerably. This mainly because irregularities of the ionospheric plasma density can cause rapid amplitude and random phase variations of satellite navigation signals (Yasyukevich et al. 2021). Even so, the convergence behavior of the IW-RTK model, especially for the horizontal components, is superior to that of the RTK model, which means that the between-station single-differenced ionospheric pseudo-observations can still reduce the convergence time during the high ionospheric disturbance. And fast PAR is still achieved in the PPPRTK case. In addition, the convergence behavior of the float solutions for the PPP-RTK case is still slightly worse than those of the other three cases after the PPP-RTK float solutions converge to $2 \mathrm{~cm}$ and $20 \mathrm{~cm}$ for the east and up components.

As listed in Table 9, in the PPP-RTK case, it takes approximately $25 \mathrm{~min}$ to converge to $2 \mathrm{~cm}$ and $5 \mathrm{~cm}$ for the horizontal and vertical components, respectively, which is almost triple the convergence time during the low solar activity days. According to the mean convergence behavior, the mean horizontal positioning errors still converge to $10 \mathrm{~cm}$ at the first epoch during the high solar activity days in the PPP-RTK case. The difference is that the degree of improvement in the convergence behavior from float- to fixed-ambiguity solutions in the east component during the high solar activity days is less than that during the low solar activity days for all cases due to the influence of the high ionospheric disturbance.

Table 10 shows the mean RMS values, TTFFs, and fixed rates during the high solar activity days. In the PPP-RTK case, the mean RMS values of the east and up components increase by approximately double during the high solar activity days compared with those during the low solar activity days. But the mean RMS values are still below $1 \mathrm{~cm}$ on the horizontal components and are approximately $2.6 \mathrm{~cm}$ on the up component. The mean TTFF is approximately $16.8 \mathrm{~min}$, which means that approximately 34 epochs are needed to fix more than $60 \%$ of all the float ambiguities to integers. Moreover, more than $90.6 \%$ of all the epochs have
Table 9 Mean convergence time for the thresholds of $2 \mathrm{~cm}$, $5 \mathrm{~cm}$, and $10 \mathrm{~cm}$ for all the 4-h sessions of the 25 user stations on days $251 / 252 / 253$ in 2014

\begin{tabular}{|c|c|c|c|c|c|c|c|c|}
\hline \multirow[b]{4}{*}{ PPP-RTK } & \multicolumn{3}{|c|}{ East (min) } & \multicolumn{3}{|c|}{ North (min) } & \multicolumn{2}{|c|}{$\mathrm{Up}(\min )$} \\
\hline & $2 \mathrm{~cm}$ & $5 \mathrm{~cm}$ & $10 \mathrm{~cm}$ & $2 \mathrm{~cm}$ & $5 \mathrm{~cm}$ & $10 \mathrm{~cm}$ & $5 \mathrm{~cm}$ & $10 \mathrm{~cm}$ \\
\hline & \multicolumn{8}{|l|}{ Float } \\
\hline & 101.5 & 3.5 & 0.5 & 35.5 & 6.5 & 0.5 & 51.5 & 17.0 \\
\hline PPP-AR & 118.5 & 70.5 & 28.0 & 74.0 & 30.0 & 8.5 & 62.0 & 32.0 \\
\hline IW-RTK & 104.5 & 14.5 & 1.5 & 54.5 & 9.0 & 2.0 & 45.0 & 14.5 \\
\hline RTK & 101.5 & 48.5 & 12.0 & 60.5 & 18.5 & 7.0 & 49.5 & 27.5 \\
\hline \multicolumn{9}{|l|}{ Fixed } \\
\hline PPP-RTK & 14.0 & 4.0 & 0.5 & 16.5 & 5.5 & 0.5 & 25.0 & 12.0 \\
\hline PPP-AR & 73.0 & 40.0 & 15.5 & 46.5 & 30.5 & 8.5 & 43.0 & 31.5 \\
\hline IW-RTK & 48.5 & 12.0 & 1.5 & 28.5 & 8.5 & 2.0 & 33.0 & 12.0 \\
\hline RTK & 61.5 & 34.0 & 12.0 & 43.0 & 18.0 & 7.0 & 39.0 & 26.5 \\
\hline
\end{tabular}


Table 10 Mean RMS values, TTFFs, and fixed rates for all the 4-h sessions of the 25 user stations on days 251/252/253 in 2014

\begin{tabular}{llllll}
\hline & \multicolumn{2}{l}{$\begin{array}{l}\text { Overall mean RMS } \\
(>90 \text { min, unit: } \mathrm{cm})\end{array}$} & & TTFF (min) & $P_{\text {fixed }}(\%)$ \\
\cline { 2 - 4 } & East & North & Up & & \\
\hline PPP-RTK & 0.8 & 0.8 & 2.6 & 16.8 & 90.6 \\
PPP-AR & 1.1 & 1.1 & 2.6 & 53.4 & 75.3 \\
IW-RTK & 0.9 & 0.9 & 2.4 & 44.9 & 80.9 \\
RTK & 1.0 & 0.9 & 2.4 & 58.5 & 75.1 \\
\hline
\end{tabular}

high-accuracy user positions. The positioning performance of the PPP-RTK model has been decreased considerably also because the applied spatial interpolation model may not be suitable for the real ionospheric state during storm-level ionospheric activity (Próchniewicz and Walo 2012). In four PAR cases, the mean RMS values of the east component, which usually reflects the performance of the estimation and fixing of ambiguities, are most affected by the high ionospheric disturbance and are double as those during low solar activity days.

\section{Conclusions}

This study first developed an IW UDUC PPP-RTK network model from the UDUC PPP-RTK network model described by Odijk et al. (2016). The between-station single-differenced ionospheric pseudo-observations strengthen the structure of the IW UDUC PPP-RTK network model. In addition, only the pivot receiver DCB, instead of the different receiver DCBs, is included in the network ionospheric corrections, which as a consequence facilitates the ionospheric interpolation at the user side.

Second, the kinematic positioning performance of the PPP-RTK model is analyzed during the low solar activity days. The corresponding results are as follows:

- It takes less than 20 epochs for the positioning errors to converge to the thresholds of $2 \mathrm{~cm}$ and $5 \mathrm{~cm}$ for the horizontal and vertical components, respectively, which reduces the convergence time on the east component by 92\% from the float- to fixed-ambiguity solutions.

- The mean TTFF is approximately 20.8 epochs, and the mean fixed rate is $95.5 \%$. After the initialization of 20 epochs, the mean RMS values of the positioning errors are $0.47,0.58$, and $1.66 \mathrm{~cm}$ for the east, north, and up components, respectively.

Third, the PPP-AR, RTK, and IW-RTK models are analyzed and compared with the PPP-RTK model to further show the better positioning performance of the latter. The corresponding results are as follows:

- The positioning performance of the PPP-RTK model is superior to those of the other three models since the PPPRTK model is constrained by network-derived precise ionospheric corrections;

- The four models can be ordered by their positioning performances as follows: PPP-RTK $>$ IW-RTK $>$ PPP-AR $>$ RTK. The positioning accuracies for the four models are similar after a long observation time span, for example, 90 epochs in this study;

Finally, to analyze the influence of the high ionospheric disturbance, the positioning performances are further analyzed during the high solar activity days. The corresponding results are as follows:

- In the PPP-RTK model, the mean RMS values of the east and up components increase by approximately double, and the mean TTFF increases by $61.5 \%$ due to the influence of the high ionospheric disturbance. However, accuracies below $1 \mathrm{~cm}$ for the horizontal direction and approximately $2.6 \mathrm{~cm}$ for the vertical direction are achieved within approximately 34 epochs.

Acknowledgements This work was partially funded by the National Natural Science Foundation of China (Grant Nos. 42022025, 41774042), the Key Research and Development Plan of Hubei Province (Grant No. 2020BHB014) and the Scientific Instrument Developing Project of the Chinese Academy of Sciences (Grant No. YJKYYQ20190063). The second author is supported by the CAS Pioneer Hundred Talents Program.

Data availability GPS code and phase measurements can be downloaded from the NOAA's national geodetic survey (NGS) (ftp://geode sy.noaa.gov/cors/rinex/). The precise orbit products have been downloaded from the CODE (ftp://ftp.aiub.unibe.ch/CODE/). The priori known station coordinates are calculated by the CSRS-PPP online application of Natural Resources Canada. Also, the sunspot number can be obtained from the http://sidc.be/silso/datafiles, and the information of the solar flare can be obtained from https://www.lmsal.com/ solarsoft/latest_events_archive.html.

Open Access This article is licensed under a Creative Commons Attribution 4.0 International License, which permits use, sharing, adaptation, distribution and reproduction in any medium or format, as long as you give appropriate credit to the original author(s) and the source, provide a link to the Creative Commons licence, and indicate if changes were made. The images or other third party material in this article are included in the article's Creative Commons licence, unless indicated otherwise in a credit line to the material. If material is not included in the article's Creative Commons licence and your intended use is not permitted by statutory regulation or exceeds the permitted use, you will need to obtain permission directly from the copyright holder. To view a copy of this licence, visit http://creativecommons.org/licenses/by/4.0/. 


\section{References}

Blewitt G (1989) Carrier phase ambiguity resolution for the global positioning system applied to geodetic baselines up to $2000 \mathrm{~km}$. J Geophys Res: Solid Earth 94(B8):10187-10203

Collins P, Lahaye F, Heroux P, Bisnath S (2008) Precise point positioning with ambiguity resolution using the decoupled clock model. Proc: ION GNSS 2008, Institute of Navigation, Savannah, Georgia,USA, September 16-19, 1315-1322

Ge M, Gendt G, Rothacher M, Shi C, Liu J (2008) Resolution of GPS carrier-phase ambiguities in precise point positioning (PPP) with daily observations. J Geodesy 82(7):389-399

Laurichesse D, Mercier F, Berthias JP, Broca P, Cerri L (2009) Integer ambiguity resolution on undifferenced GPS phase measurements and its application to PPP and satellite precise orbit determination. Navigation 56(2):135-149

Li X, Zhang X, Ge M (2011) Regional reference network augmented precise point positioning for instantaneous ambiguity resolution. J Geodesy 85(3):151-158

Li L, Zhang D, Zhang SR, Coster AJ, Hao YQ, Xiao Z (2015) Influences of the day-night differences of ionospheric variability on the estimation of GPS differential code bias. Radio Sci 50(4):339-353

Li M, Yuan Y, Wang N, Liu T, Chen Y (2017) Estimation and analysis of Galileo differential code biases. J Geodesy 91(3):279-293

Malys S, Jensen PA (1990) Geodetic point positioning with GPS carrier beat phase data from the CASA UNO experiment. Geophys Res Lett 17(5):651-654

Odijk D (2002) Fast precise GPS positioning in the presence of ionospheric delays. Dissertation, Netherlands Geodetic Commission, Delft

Odijk D, Verhagen S (2007) Recursive detection, identification and adaptation of model errors for reliable high-precision GNSS positioning and attitude determination. Proc: 2007 IEEE RAST, Istanbul, Turkey, June 14-16, 624-629

Odijk D, Zhang B, Khodabandeh A, Odolinski R, Teunissen P (2016) On the estimability of parameters in undifferenced, uncombined GNSS network and PPP-RTK user models by means of S-system theory. J Geodesy 90(1):15-44

Odolinski R, Teunissen P, Odijk D (2015) Combined GPS+ BDS for short to long baseline RTK positioning. Measurement Sci Technol 26(4):045801

Olea R.A. (1999) Ordinary Kriging. In: Geostatistics for Engineers and Earth Scientists. Springer, Boston, MA. https://doi.org/10. 1007/978-1-4615-5001-3_4

Próchniewicz D, Walo J (2012) Quality indicator for ionospheric biases interpolation in the Network RTK. Reports Geodesy 92(1):7-21

Psychas D, Verhagen S (2020) Real-time PPP-RTK performance analysis using ionospheric corrections from multi-scale network configurations. Sensors 20(11):3012

Psychas D, Verhagen S, Liu X, Memarzadeh Y, Visser H (2018) Assessment of ionospheric corrections for PPP-RTK using regional ionosphere modelling. Measurement Science and Technology, 30(1):014001

Rocken C, Ware R, Van Hove T, Solheim F, Alber C, Johnson J, Bevis M, Businger S (1993) Sensing atmospheric water vapor with the global positioning system. Geophys Res Lett 20(23):2631-2634

Teunissen P (1995) The least-squares ambiguity decorrelation adjustment: a method for fast GPS integer ambiguity estimation. $\mathbf{J}$ Geodesy 70:65-82

Teunissen P, Khodabandeh A (2013) BLUE, BLUP and the Kalman filter: some new results. J Geodesy 87(5):461-473
Teunissen P, Montenbruck O (2017) Springer handbook of global navigation satellite systems. Springer International Publishing, Switzerland

Teunissen P, Verhagen S (2009) The GNSS ambiguity ratio-test revisited: a better way of using it. Surv Rev 41(312):138-151

Teunissen P, Odijk D, Zhang B (2010) PPP-RTK: results of CORS network-based PPP with integer ambiguity resolution. J Aeronaut, Astronaut Aviat 42(4):223-230

Van Bree R, Tiberius C, Hauschild A (2009) Real time satellite clocks in single-frequency precise point positioning. Proc: ION GNSS 2009, Institute of Navigation, Savannah, Georgia, USA, September $22-25,2400-2414$

Wang K, Khodabandeh A, Teunissen P (2017) A study on predicting network corrections in PPP-RTK processing. Adv Space Res 60(7): 1463-1477

Wübbena G, Schmitz M, Bagge A (2005) PPP-RTK: precise point positioning using state-space representation in RTK networks. Proc: ION GNSS 2005, Institute of Navigation, Long Beach, Los Angeles, USA, September 13-16, 2584-2594

Yasyukevich YV, Yasyukevich AS, Astafyeva EI (2021) How modernized and strengthened GPS signals enhance the system performance during solar radio bursts. GPS Solutions 25(2):1-12

Zumberge J, Heflin M, Jefferson DC, Watkins MM, Webb FH (1997) Precise point positioning for the efficient and robust analysis of GPS data from large networks. J Geophys Res: Solid Earth 102(B3):5005-5017

Publisher's Note Springer Nature remains neutral with regard to jurisdictional claims in published maps and institutional affiliations.

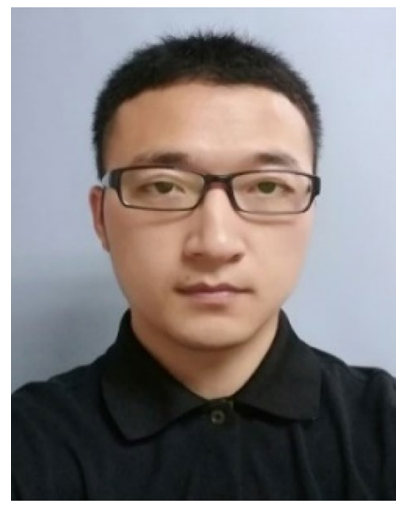

Jiuping Zha received his Ph.D. at the Innovation Academy for Precision Measurement Science and Technology, CAS, in July 2021, where he is now an assistant researcher. His current research interests include multiGNSS integer ambiguity resolution and enable precise point positioning (PPP-RTK) algorithms and related applications.

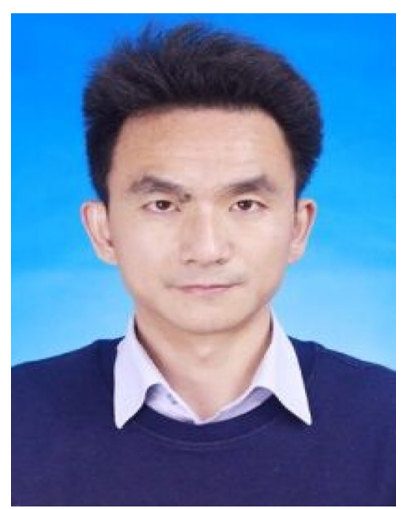

Baocheng Zhang is a professor at the Innovation Academy for Precision Measurement Science and Technology, CAS. His research focuses on modeling multiple global navigation satellite systems for integer ambiguity resolution-enabled precise point positioning (PPP-RTK) applications. 


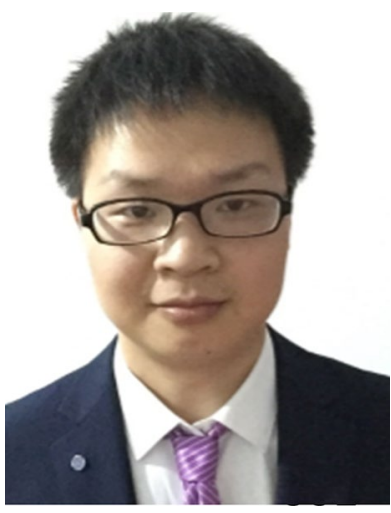

Teng Liu is an associate researcher at the Innovation Academy for Precision Measurement Science and Technology, CAS. His research focuses on the models and algorithms of multiGNSS and multi-frequency PPPRTK and related applications.

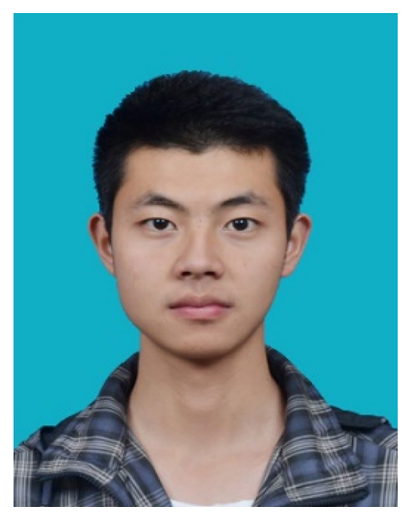

Pengyu Hou is a Ph.D. candidate at the Innovation Academy for Precision Measurement Science and Technology, CAS. His current research focuses on multiGNSS integer ambiguity resolution-enabled precise point positioning (PPP-RTK), with a great interest in the GLONASS system. 